\title{
DiAgNOSTICAR PARA IMPLEMENTAR EN Educación Matemática
}

\author{
Diagnosing to Implement in Mathematical Education
}

\author{
Oscar Alemany Llanos \\ Especialista en Metodología Matemática \\ Colegio Alcántara La Florida \\ Vicuña Mackenna Oriente 6081, La Florida \\ oscaralemany@yahoo.es
}

Recibido: 26 de julio, 2007. Aceptado: 28 de agosto, 2007

\begin{abstract}
Resumen: El trabajo que se presenta muestra una propuesta de asistencia en el área de educación matemática para alumnos de enseñanza media. Para su implementación se consideró, por una parte, la aplicación de técnicas de naturaleza cualitativa como medio para diagnosticar en profundidad la situación que ellos presentan en esta asignatura; por otra, los datos recogidos de talleres realizados con la población de alumnos que en el estudio se diagnosticó en situación de riesgo, centrando el trabajo en aula en la definición y construcción de conceptos y de aquellos subyacentes a ellos asociados a los temas tratados, y en el uso de metodologías que enfatizan la noción de significado y la negociación de significados de las materias tratadas.
\end{abstract}

Palabras clave: Conceptos matemáticos, negociación de significados, diagnosticar en profundidad, desesperanza aprendida, entrevista matemática.

Abstract: The present work is a proposal of pedagogical assistance in the field of mathematical education for students in secondary education. Its implementation considered, on one hand, the application of techniques of a qualitative nature as a way to diagnose in depth the situation they present in this subject; on the other hand, the information gathered from workshops with the students diagnosed in the study in situation of risk, centring the classroom work on the definition and construction of concepts and of those sublying them associated with the topics treated, and on the use of methodologies that emphasize the notion of meaning and the negotiation of meanings on the issues treated.

Key words: Mathematical concepts, negotiation of meanings, diagnose in depth, learned despair, mathematical interview.

\section{INTRODUCCIÓN}

En la actualidad, la enseñanza de la matemática en nuestros colegios es concebida como una transmisión de contenidos que se trabajan, usualmente, 
a partir de la definición y de la ejercitación mecánica de los conceptos asociados a ellos desde la perspectiva del profesor y de los equipos técnicos del que forma parte; omiten en sus exposiciones los conceptos que sustentan los conceptos definidos y la construcción exhaustiva de éstos; sin considerar, en este proceso, el significado que los alumnos les asignan. Por tanto, se desconoce la situación en profundidad presentada por ellos en esta asignatura.

Esto tiene como consecuencia, en primer lugar, que estos equipos planifiquen la enseñanza de la matemática a partir de datos parciales acerca del nivel que tienen los alumnos, usualmente obtenidos a raíz de pruebas, que son tomadas como diagnóstico, donde se mide el conocimiento que tienen de determinadas materias; y en segundo lugar, que los alumnos se vean en la imposibilidad de comprender los temas expuestos cuando los conceptos que forman parte de la cadena del concepto que define el tema no estén plenamente incorporados a su sistema cognitivo.

Este es un estudio de casos realizado con alumnos de enseñanza media de colegios subvencionados de la Región Metropolitana en el que se investigó el proceso de enseñanza y aprendizaje de las matemáticas. El significado que estos alumnos le dan a este proceso, y la noción de significado que se estudió desde la perspectiva de la educación matemática, permitieron diagnosticar en profundidad la situación que ellos presentan en esta área. Sobre la base de los datos obtenidos se estructuró una metodología centrada en la noción y negociación de significados matemáticos que se aplicó en talleres dirigidos a la población de alumnos diagnosticados en situación de riesgo. La información que emanó de estos talleres permitió elaborar planes de asistencia pedagógica en educación matemática destinados a este tipo de alumnos, donde se enfatiza la construcción en profundidad de los conceptos asociados a los temas tratados y la revisión de la cadena de conceptos del que forman parte.

\section{APROXIMACIONES TEÓRICAS Y CONSIDERACIONES METODOLÓGICAS}

Este estudio abarcó un universo de aproximadamente 800 alumnos con rendimiento alto, medio y bajo en la asignatura de matemática de siete primeros medios, siete segundos medios, y seis terceros medios de dos colegios subvencionados de la Región Metropolitana clasificados en el grupo socioeconómico medio alto. 
Para conocer el significado que los alumnos del estudio dan al proceso de enseñanza y aprendizaje de la matemática, la investigación se diseñó desde una perspectiva metodológica de tipo cualitativa, utilizando como técnicas para la recolección de datos, la observación, el grupo focal, la entrevista, y la observación participante.

Se convocó a 60 grupos focales constituidos por 8 alumnos de rendimiento bajo, medio y alto de cada uno de los cursos del estudio, en total 480 alumnos. Para saturar el universo simbólico de significados en relación al fenómeno en estudio, se hizo una serie de entrevistas a alumnos de estos cursos, algunas de ellas en profundidad, observaciones de clases, y se dictó clases de matemática a cada uno de estos cursos con el propósito de conocer el significado que, para el conjunto de los alumnos tenían los conceptos matemáticos asociados a los contenidos que están dentro del marco curricular de esta asignatura.

El proceso de triangulación se realizó mediante entrevistas individuales a profesores jefes de los cursos del estudio y profesores de matemática, no todos de los cursos estudiados, y a directivos.

El análisis de los datos obtenidos, tendiente a develar el significado que tenía para los alumnos del estudio el proceso de enseñanza y aprendizaje de las matemáticas, no mostró el problema matemático que gatilla la situación de desesperanza que experimentan los alumnos sobre su fracaso escolar.

En consecuencia, en el transcurso de la investigación, se hizo necesario buscar una técnica investigativa que permitiera conocer qué elementos matemáticos gatillaban esta situación. Al entrevistar sobre problemas matemáticos a algunos alumnos de rendimiento bajo del estudio, se obtuvo una herramienta que podía dar respuesta acerca de qué aspectos de la matemática producían esa situación de frustración, y más aún, si era posible revertirla y cómo.

Esta técnica que denominamos Entrevista Matemática se utilizó también en alumnos del estudio con otros niveles de rendimiento, y al conocer la interpretación que alumnos de altas calificaciones hicieron de su nivel de conocimiento, comprensión, aplicación y análisis de conceptos matemáticos, se constató que sus discursos no coincidieron con el nivel de las habilidades que exhibieron ante distintas situaciones matemáticas que se les propusieron resolver. La entrevista matemática se aplicó a los 60 grupos de alumnos que integraron los grupos focales, cuyos datos permitieron conocer el significado 
que para estos alumnos tiene el proceso de enseñanza - aprendizaje de la matemática y que, específicamente, midió el nivel:

a) que estos alumnos tienen para operar con representaciones materiales ostensivas;

b) que estos alumnos tienen para aplicar conceptos matemáticos a situaciones-problema en contextos matemáticos y de la vida cotidiana;

c) de logro que estos alumnos tienen en cuanto a alcanzar generalizaciones, abstracciones e ideas matemáticas.

Los datos obtenidos a través de esta técnica permitieron diagnosticar la situación que presentan estos alumnos en la asignatura de matemática, y en particular, conocer la población de alumnos en situación de riesgo que formaría parte de los talleres. En talleres se trabajó el significado del conocimiento matemático a través de la relación entre los signos usados para codificarlo y los contextos que lo establecen. Dado que en el trabajo matemático tanto las ideas matemáticas, sus abstracciones, como las situaciones-problema están representadas por sistemas notacionales que las describen, el trabajo de la parte conceptual realizado se relaciona con la teoría de los campos conceptuales de Vergnaud (1982), en la que un concepto es la unión del conjunto de situaciones que lo hacen significativo, así como el conjunto de invariantes que lo constituyen y el conjunto de representaciones simbólicas que se usan para presentarlo, para dar sus propiedades y las situaciones a las que se refiere.

Para construir los conceptos matemáticos se utilizó la perspectiva teórica de Duval (1995), que señala que la construcción de conceptos matemáticos no basta hacerla dentro de un solo sistema de representación, dado que cada representación es parcial con respecto al concepto que representa, sino que se debe considerar como absolutamente necesaria la interacción entre diferentes representaciones del objeto matemático para su formación, debiendo realizarse las tareas de conversión de una representación a otra, y viceversa. Son éstas las que propiciarían la construcción de conceptos matemáticos.

El conocimiento matemático se modeló a partir del esquema de Steinbring (1997), quien incluye los conceptos, los signos/símbolos y los objetos/contexto de referencia como elementos que lo interpretan; la construcción individual de los significados matemáticos se hizo desde la perspectiva del interaccionismo simbólico.

Según Cobb y Bauersfed (1995), en este enfoque de investigación, esta construcción tiene lugar en la interacción con la cultura de la clase, y al 
mismo tiempo contribuye a la constitución de esta cultura. Así, el aprendizaje matemático es un proceso personal, por una parte, y por otra, un proceso de adaptación a una cultura de manera interactiva, más que una internalización de normas y conocimiento objetivado. Según Bauersfeld (1994), la práctica matemática en el aula es un proceso de matematización compartida que define una subcultura específica para ese profesor, esos alumnos y esa aula.

La negociación de los significados matemáticos es relevante en el proceso de formación del concepto en el aula, relevancia que se manifiesta al observar cuidadosamente los microprocesos que ocurren en una clase.

De esta manera la negociación de los significados matemáticos es una forma de describir el modo en que los estudiantes desarrollan la comprensión de los conceptos matemáticos, obteniendo creencias y actitudes acordes con el sentido que tienen las matemáticas.

\section{RESULTADOS}

\section{Significado que dan los alumnos del estudio al proceso de enseñanza-aprendizaje de las Matemáticas}

\subsection{Alumnos de nivel bajo}

A los alumnos de bajo rendimiento en matemática no les gusta la matemática, la encuentran complicada, les es difícil, la estudian por obligación. Estos alumnos sienten inseguridad frente a las pruebas y desmotivación constante.

Tienen profesores que utilizan una metodología que no considera la presentación de los conceptos que subyacen al concepto que se está enseñando, y que no consideran las distintas combinaciones entre ellos, que los obligan a poner atención a la resolución de ejercicios centrados en la sintaxis del lenguaje simbólico matemático más que en la semántica de la actividad matemática.

Para estos alumnos, la clase de matemática significa una obligación que deben cumplir, teniendo que soportar a un profesor que dirige la clase hacia los mejores alumnos, que los ignora sólo por tener un ritmo de aprendizaje más lento; esto les significa un estado de tensión constante con el profesor, lo que se agudiza cuando le piden que repase los conceptos que tienen olvidados o que nunca internalizaron. 
Todas las situaciones anteriores por las que atraviesan los alumnos con fracaso escolar producen en ellos un estado que el psicólogo Martín Seligman (1975) denominó como desesperanza aprendida (DA). El estudiante que está en este estado siente que cualquier esfuerzo que haga por revertir su bajo nivel en matemática, de nada servirá. Él plantea que la indefensión de las personas deprimidas (caracterizada por no tratar de evitar los acontecimientos negativos) es un factor primario de la depresión, en sí misma, es un modelo aprendido de conducta. Denomina a este modelo de condicionamiento operante inefectivo, desesperanza aprendida o indefensión aprendida.

El sugestivo paralelismo con el estado de DA que guarda la percepción de los alumnos en situación de fracaso escolar en la asignatura de matemática sobre las circunstancias y vicisitudes por las que atraviesan, permite comprender las distorsiones cognitivas que le impiden ver la salida a su problema para lograr un afrontamiento más positivo de su situación, siendo éste el significado que dan al proceso de enseñanza y aprendizaje de las matemáticas.

\subsection{Alumnos de nivel medio y de nivel avanzado}

A los alumnos de rendimiento medio, la clase de matemática les significa el tener que ocupar parte de sus vidas en desarrollar actividades vinculadas a contenidos que no entienden de dónde vienen y dónde se aplican. Actividades que tienen que realizar para alcanzar una nota que, por una parte, les evite las molestias ocasionadas por las constantes discusiones que sobre su rendimiento tienen con sus padres, y por otra, contribuyan a alcanzar un NEM ( notas de la enseñanza media ) que les permita postular a una carrera de su preferencia en la universidad.

A los alumnos de rendimiento alto, la clase de matemática les significa el realizar actividades centradas en el trabajo con representaciones materiales ostensivas de manera mecánica, teniendo que poner atención a un profesor estructurado, que repite el mismo tipo de explicaciones de manera frecuente, y donde las situaciones-problema y las aplicaciones se limitan al contexto de fórmulas y teoremas de los que no explica su origen y sus generalizaciones, no existiendo, de esta manera, la posibilidad de que estos alumnos conozcan la génesis y las abstracciones del conocimiento matemático, y su aplicación en contextos de la vida cotidiana. 


\section{Nivel Matemático que presentan los alumnos del estudio y factores principales que inciden en él}

De las entidades básicas medidas, los alumnos de nivel matemático bajo sólo logran parte de la notacional, es decir, operan en cierto grado con términos, expresiones, gráficos, símbolos, etc.

Los alumnos de nivel matemático medio logran, en parte, resolver actividades que involucran notaciones, haciendo aplicaciones básicas en contextos matemáticos y de la vida cotidiana, pero no logran generalizar resultados obtenidos en situaciones particulares, hacer abstracciones, y generar ideas matemáticas como resultado de su trabajo.

Aquellos de alto rendimiento operan de manera satisfactoria todo tipo de representaciones materiales ostensivas, logran hacer aplicaciones de niveles básicos y medios en contextos matemáticos y cotidianos y sólo generalizan situaciones particulares elementales asociadas al lenguaje algebraico, no logrando hacer abstracciones ni concebir ideas matemáticas.

\section{TALLERES REALIZADOS CON ALUMNOS DE RENDIMIENTO BAJO Y ALTO EN MATEMÁTICA}

Los datos obtenidos del proceso anteriormente descrito permitieron diagnosticar en profundidad la situación que presentan estos alumnos en la asignatura. En particular, permitieron detectar que las poblaciones en situación de riesgo en este ramo son las que congregan a los alumnos de niveles de bajo y alto rendimiento.

Se desarrollaron dos talleres con alumnos de nivel bajo y un taller con alumnos de nivel alto.

Los seis alumnos de nivel bajo que integraron los talleres, fueron seleccionados por tener los rendimientos más bajos en la asignatura de matemática en sus cursos de segundos medios en los dos colegios estudiados; los de nivel alto, y cuyo número fue también de seis, correspondieron a los alumnos de más alto rendimiento seleccionados de los segundos y terceros medios de uno de los colegios del estudio.

Antes de iniciar la investigación partimos del supuesto de que la población de mayor rendimiento en matemática sólo necesitaba un mínimo de esfuerzo para ingresar a carreras de su preferencia en la universidad; pero este estudio 
mostró que el nivel que ellos dicen tener no corresponde al que se necesita para cumplir su objetivo, más aún, no tienen las conductas intensivas básicas para entender las materias de los ramos matemáticos de los primeros cursos universitarios; de esta manera, se pueden considerar en situación de riesgo si postulan su ingreso a este tipo de carreras y si pretenden permanecer en ellas.

Respecto a los alumnos de rendimiento bajo, el estudio ratifica su situación de riesgo con respecto a su ingreso a la universidad, pues el nivel básico que tienen les impediría obtener el puntaje necesario en la prueba de selección universitaria (PSU) matemática para alcanzar su meta.

En cambio, el análisis de los datos obtenidos de los alumnos de rendimiento medio, devela, por una parte, que la mayoría de ellos tiene intereses que se orientan hacia carreras de exigencia menor en esta área; y por otra, que el nivel que presentan en la operatoria con representaciones materiales ostensivas y en las entidades extensionales que inducen la actividad matemática, les permitiría obtener en la PSU matemática el puntaje necesario para ingresar a carreras con ese nivel de exigencia.

\section{$\underline{\text { Talleres con alumnos de nivel bajo }}$}

El contenido trabajado, en quince sesiones de una hora y media cada una, fue la ecuación de la recta en el plano cartesiano.

Después de diagnosticar la situación que presentan estos alumnos respecto de los elementos que constituyen esta ecuación, se trabaja este concepto utilizando la tripleta que propone la teoría de los campos conceptuales de Vergnaud. Al respecto, los alumnos lograron, a partir de una serie de actividades a las que se enfrentaron, conocer el origen y las utilidades que tiene este objeto en contextos que son de su interés, es decir, trabajaron en el conjunto de situaciones que lo hacen significativo.

La construcción de los invariantes que constituyen este concepto se hace a partir de la definición de éstos. Se realiza teniendo presente que lo importante de esta etapa es que el alumno adquiera el concepto que se está definiendo y que se logra cuando una imagen de él se ha incorporado a su bagaje mental. Los alumnos lograron construir este concepto cuando los elementos que forman parte de la cadena asociada a él, como lo son los ejes coordenados, pendiente, coeficiente de posición y otros que intervienen en la ecuación de la recta, los trabajaron desde diferentes sistemas de representación. La interacción que establecieron entre estos sistemas, por ejemplo, las de algunas 
visualizaciones con sus representaciones algebraicas, facilitaron la comprensión de esta ecuación. Es así como la construcción que hicieron de las imágenes de triángulos rectángulos que tenían como vértices a puntos pertenecientes a rectas en un computador, les permitieron la visualización de sus pendientes, las que contrastadas con las fórmulas que las definieron, produjeron la plena internalización del concepto.

El trabajo de los alumnos con las representaciones simbólicas usadas para presentar el concepto les permitió adquirir técnicas de cálculo para operar con los términos, expresiones y símbolos que le dan corporeidad. Las situaciones-problema y las abstracciones matemáticas a que los alumnos se enfrentaron, en particular los problemas de aplicación resueltos y propuestos en el taller, sólo se pudieron concretar cuando ellos exhibieron cierta destreza en la operatoria con las representaciones materiales ostensivas que les dan forma.

Una parte fundamental en la metodología usada fue la negociación de los significados de los conceptos involucrados en el tema tratado que hicieron los alumnos y el profesor de los talleres. Cuando se introdujeron esos conceptos, se dio una situación plurisemántica, es decir, el significado que el profesor les otorgaba era distinto de lo que estos objetos significaban para cada uno de los estudiantes.

La metodología en referencia dio lugar a que los alumnos aunaran los significados que cada uno de ellos le atribuía a los conceptos vinculados a la ecuación de la recta, obteniendo un significado compartido de pendiente, coeficiente de posición, etc.

Otro aspecto trabajado fue la 'reparación' de aquellos conceptos y técnicas que los estudiantes no tenían claros y de repasar aquello que ellos no recordaban, por ejemplo, el concepto de variable independiente y dependiente, el concepto triángulos semejantes, la técnica de despejar una incógnita en una ecuación, etc. De hecho, en todas las actividades planteadas, se colocó a disposición de los alumnos los conceptos que se necesitaban para trabajarlos.

La metodología anterior fue un elemento que facilitó el trabajo de los alumnos, en efecto, los conceptos que aparecieron en los enunciados de las situaciones-problema a los que se vieron enfrentados no fueron un obstáculo en el modelamiento y posterior resolución de la ecuación resultante de este proceso, incluso del análisis y de la proyección de los resultados obtenidos, pues siempre los tuvieron a mano. 
La satisfacción que produjo en ellos el saber de qué se trata un problema y de tener a su disposición los conceptos necesarios para resolverlo, fueron un elemento clave para que estos alumnos terminaran con su estado de desesperanza aprendida, pues se enfrentaron con éxito a problemas que antes les era impensado resolver; es más, algunos de ellos decidieron tomar electivos matemáticos cuando cursaran el tercer año medio, pues sentían que la matemática ya no les era esquiva. Este es el principal resultado del trabajo realizado con estos alumnos.

Todo este proceso se facilitó por la manera en que se estructuraron las clases. Al inicio de cada sesión, profesor y alumnos discutieron las actividades propuestas para la casa y las actividades de la clase anterior que no fueron resueltas por la totalidad de los estudiantes; a continuación se presentaron las situaciones, no más de tres, que debían ser trabajadas en el taller. Éstas fueron, en un comienzo, intentadas resolver por cada estudiante de manera individual para posteriormente, después de una discusión en la que se negociaban los significados y se daban a conocer las posturas de cada uno de ellos, resolverlas por el conjunto de los alumnos. En todo este proceso el profesor fue un mediador del aprendizaje que puso a disposición de los estudiantes los conceptos que se necesitaban para entender estas situaciones. Estos problemas requirieron del alumno el trabajo con conceptos, con signos/ símbolos y con objetos/contexto de referencia, lo que permitió que ellos lograran, en niveles básicos y medios, técnicas con representaciones materiales ostensivas, realizar aplicaciones en contextos cotidianos y obtener generalizaciones y abstracciones matemáticas.

\section{Talleres con alumnos de nivel alto}

Con estos alumnos se trabajó en la resolución de 200 problemas de geometría plana durante doce sesiones de una hora y media cada una. Estos problemas exigen del estudiante un manejo notacional elevado, un grado de nivel medio-avanzado de aplicación de la geometría en contextos matemáticos, y un grado de nivel medio en las entidades intensionales en geometría.

Una vez conocido el significado, a través de entrevistas matemáticas, que estos alumnos le dan a la geometría y el nivel que ellos presentan en esta área, se les propone la resolución de estos problemas.

El trabajo en el taller se inició con la exhibición de los teoremas asociados a los contenidos involucrados en la geometría plana. 
La primera tarea que tuvieron que realizar los alumnos fue la construcción de los conceptos geométricos que dan forma a estos teoremas; a continuación se les pidió la demostración de estos últimos.

Después de trabajar diferentes representaciones del objeto matemático para su formación, realizando las tareas de conversión de una representación a otra, y viceversa, los alumnos lograron la construcción de estos conceptos.

El trabajo que hicieron con los sistemas notacionales que dan forma a estas estructuras matemáticas, les permitió organizar los elementos de manera adecuada para lograr la segunda meta. A modo de ejemplo, los alumnos demostraron la relación que existe entre el ángulo comprendido entre dos cuerdas de una circunferencia con sus arcos, es decir, demostraron que el ángulo pedido corresponde a la semisuma de los arcos comprendidos entre esas cuerdas, luego de construir los conceptos de circunferencia, de cuerda y de arco de una circunferencia, y trabajar con las notaciones que los representan.

Realizadas las tareas anteriores los alumnos trabajaron los problemas propuestos. A diferencia de los talleres efectuados con los alumnos de bajo rendimiento, estos alumnos trabajaron individualmente los problemas, consultándose algunas veces entre ellos, pero sin establecer una comunicación con el profesor del taller para hacer alguna pregunta relativa a las situaciones que resolvieron.

Revisadas las resoluciones de los problemas propuestos se concluyó que estos alumnos modelan el conocimiento geométrico a través del esquema que incluye los conceptos, los signos/símbolos y los objetos/contexto de referencia como elementos que lo interpretan.

Estos estudiantes expresan que los problemas que les ocasionaron mayor dificultad fueron aquellos en los que su resolución pasaba por representar la situación propuesta en otro contexto matemático. Que para lograr la meta tuvieron que recurrir de manera constante a distintos sistemas de representación y a la interacción entre ellos. Y que la construcción de los conceptos y de aquellos que subyacen a ellos y que estaban relacionados con el problema en cuestión, más la demostración detallada de los teoremas relacionados con las situaciones planteadas, se convirtieron en elementos determinantes para alcanzar la meta propuesta. 


\section{PROPUESTA PEDAGÓGICA}

\section{1.- Para asistir a alumnos con bajo rendimiento matemático}

La propuesta que se presenta a continuación se basa en los talleres realizados con este tipo de alumnos. Consta de las siguientes actividades:

a) Desarrollar talleres de asistencia donde el número de alumnos que participen en ellos no exceda de seis; en lo posible, en lugares que no sean sus salas de clases; donde se trabajen temas específicos de manera completa de acuerdo al nivel al que pertenecen; y por un período que no exceda a los dos meses.

b) Diagnosticar la situación que presentan estos alumnos respecto de los temas que se van a tratar, utilizando para ello grupos focales y entrevista s matemáticas relativas a dichos temas.

c) Asesorar a los alumnos para que construyan los conceptos asociados a los temas que se traten a partir de distintas representaciones y a la interacción constante entre ellas.

d) Proponer a lo más tres situaciones-problema de aplicaciones en contextos cotidianos y matemáticos a desarrollar por sesión, e igual número para que sea realizado en casa, que implique un trabajo consistente en modelar estas situaciones y en manipular las entidades notacionales que surgen del proceso de modelamiento.

e) Emplear una metodología centrada en la negociación de significados, donde el profesor sea un mediador del aprendizaje y donde las soluciones de los problemas planteados sean trabajadas por el conjunto de los alumnos.

f) Poner a disposición de los estudiantes los conceptos que se necesitan para modelar y resolver las situaciones propuestas cada vez que sea necesario, revisando y clarificando aquellos que les son confusos o que no conocen relativos al tema planteado.

\section{2.-Para asistir a alumnos con alto rendimiento matemático}

La propuesta para alumnos de este rendimiento matemático incluye:

a) Realización de talleres de asistencia con cualquier número de alumnos de este nivel y en lugares que pueden ser sus salas de clases, donde se trabajen temas específicos completos de nivel avanzado, y sin límite de duración. 
b) Diagnóstico de la situación que presentan estos alumnos respecto de los temas que se van a tratar, utilizando para ello grupos focales y entrevistas matemáticas relativas a dichos temas.

El profesor, como mediador, debe asesorar las siguientes actividades para que los alumnos, proveyéndoles los conceptos y teoremas necesarios para desarrollarlas:

c) Construyan los conceptos asociados a los temas que se traten a partir de las distintas representaciones que admiten y a la interacción constante entre ellas.

d) Trabajen en las demostraciones de los teoremas asociados a los contenidos tratados.

e) Generalicen fórmulas y propiedades que surgen del modelamiento de situaciones dadas en contextos cotidianos y matemáticos.

f) Realicen actividades tendientes a desarrollar la creación y abstracción matemática.

g) Representen las situaciones propuestas en distintos marcos de referencia.

\section{EN SÍNTESIS}

Entre los factores que inciden negativamente en el desempeño de los alumnos en su actividad matemática, el estudio muestra lo siguiente:

La falta de claridad, o el desconocimiento de los conceptos involucrados en las situaciones matemáticas que se les plantean, impide a los alumnos de rendimiento bajo tener la posibilidad de hacer las relaciones necesarias para su resolución.

La falta de trabajo sistemático determina, en parte, el bajo nivel que los alumnos de rendimiento medio presentan para manipular los conceptos matemáticos relacionados con los problemas a los que tienen que enfrentarse, resolviendo sólo aquellos que requieren de técnicas básicas.

El mayor inconveniente al que se ven enfrentados los alumnos de alto rendimiento escolar en el área de matemática de acuerdo al estudio, es la cantidad insuficiente de situaciones-problema que se les han propuesto durante 
la enseñanza básica y media en esta área, que impliquen aplicaciones y generalizaciones matemáticas; esto se ve reflejado en el mal rendimiento que exhiben, por ejemplo, cuando se les proponen problemas que, para ser resueltos, requieren de su presentación en más de un marco de referencia.

El estudio realizado pretende ser un aporte y arroja como resultado que, en el caso de la educación media, una posibilidad de solución al problema de la enseñanza y aprendizaje de la matemática es:

- Diagnosticar en profundidad la situación de los alumnos de enseñanza media en el área de matemática; esto se puede hacer utilizando metodologías de naturaleza cualitativa para conocer el significado que el alumno le da a este proceso y el nivel matemático que presenta.

- Realizar talleres con la población de alumnos que estén categorizados en situación de riesgo de acuerdo al diagnóstico anterior.

- Utilizar en el aula metodologías que consideren la perspectiva del alumno en la construcción del conocimiento matemático. En el estudio se empleó la negociación de significados donde el rol del profesor es mediar el aprendizaje, y donde las soluciones de los problemas propuestos en la clase son un producto del trabajo realizado en conjunto por los alumnos.

De las entidades medidas se infiere que los alumnos del estudio resuelven situaciones problemáticas en contextos cotidianos y matemáticos sólo cuando logran establecer relaciones entre los conceptos asociados a ellas; esto implica, en primer lugar, el conocerlos, y en segundo lugar, tener las técnicas que les permita vincularlos.

Se propone que el profesor de la asignatura trabaje con estos alumnos en la clase, y de manera paralela, se propongan problemas que requieran para su resolución más trabajo con entidades intensionales que entidades notacionales, estas últimas ya adquiridas por los alumnos.

Se concluye, además, que:

El estado de desesperanza aprendida que afecta a los alumnos de bajo rendimiento escolar en el área de matemática se puede revertir. Para ello se debe trabajar desde la perspectiva de los conceptos $\mathrm{y}$, específicamente, se requiere que las situaciones-problema que se les pidan resolver estén acompañadas de la presentación de los conceptos asociados a ellas, aclarando aquellos que les sean confusos, y volviendo a tratar los que subyacen a ellos y que tienen olvidados. 


\section{REFERENCIAS BIBLIOGRÁFICAS}

Bauersfeld, H., 1994

Theoretical perspectives on interaction in the mathematics classroom. En: R. Biehler; R. Scholz; R. Strässer y B. Winkelmann (Eds.). Didactics of Mathematics as a Scientific Discipline. Dordrecht, NL: Kluwer Academic Pb. pp. 133-146.

Coob, P. y H. Bauersfeld, The emergence of mathematical meaning: 1995 Interaction in classroom cultures. Hillsdale, N. Y. Lawrence Earlbaum A.P.

Duval, R., 1995

Semiosis et penseé humaine. Registres sémiotics et apprentissages intellectuels.Peter lang. Berna.

Seligman, M., 1975

Helplessness: On depresion,Development, and Death. W. H. Freeman. San Francisco, California.

Steinbring, H., 1997

Epistemological investigation of classroom interaction in elementary mathematics teaching. En: Educational Studies in Mathematics, Vol. 32, pp. 49-52.

Vergnaud, G., 1982

Cognitive and development psychology and research in mathematics education: some theoretical and methodological issues. En: For the learning of mathematics, Vol. $3, \mathrm{~N}^{\mathrm{D}} 2$, pp 31-41. 